\title{
Espiral construtivista em cursos de graduação em educação física: ensinando sobre o Sistema Único de Saúde
}

\author{
Constructivist Spiral in the physical education undergraduate programs: teaching \\ about Unified Health System
}

\section{AUTORES \\ Braulio Nogueira de Oliveira ${ }^{1}$ (D) José Geraldo Soares Damico ${ }^{2}$ (D) \\ Alex Branco Fraga ${ }^{1,2}$ (D) \\ 1 Universidade Federal do Rio Grande do Sul, Programa de Pós-Graduacão em Ciências do Movimento Humano, Porto Alegre, Rio Grande do Sul, Brasil. \\ 2 Universidade Federal do Rio Grande do Sul, Departamento de Educação Física, Fisioterapia e Dança, Porto Alegre, Rio Grande do Sul, Brasil.}

\section{CONTATO}

Braulio Nogueira de Oliveira

brauliono08@hotmail.com

Rua Felizardo, 750, Jardim Botânico, Porto

Alegre, Rio Grande do Sul, Brasil. CEP:

90450-190.

DOI

$10.12820 /$ rbafs.23e0031

\section{cc) $(9)$}

Copyright: This is an open-access article distributed under the terms of the Creative Commons Attribution License ${ }^{\circledR}$, which permits unrestricted use, distribution, and reproduction in any medium, provided that the original author and source are credited.

\begin{abstract}
RESUMO
O objetivo deste artigo foi descrever uma experiência curricular fundamentada no referencial da espiral construtivista em uma disciplina do curso de Educação Física da Universidade Federal do Rio Grande do Sul (UFRGS), no contexto do Sistema Único de Saúde (SUS). Em quatro encontros, cada um com quatro horas-aula, em duas turmas, práticas corporais e atividades físicas (PCAFs) em interface com o SUS foram tematizadas através da elaboração e do atendimento de casos-problemas. Inspirados em situações do cotidiano de trabalho, equipes formadas pelos estudantes delimitaram e estudaram objetivos de aprendizagem a partir de necessidades emergentes. Procedimentos como a organização em rede, as tecnologias de apoio matricial, projeto terapêutico singular, uso de prontuário, genograma, ecomapa, entre outros, delimitaram o foco do trabalho de contextualização do uso das PCAF nos casos-problemas. Considerando a avaliação realizada, conclui-se que a proposta potencializou a aprendizagem dos estudantes a respeito dos saberes e práticas relativos ao SUS.
\end{abstract}

Palavras-chave: Currículo; Aprendizagem baseada em problemas; Sistema Único de Saúde; Recursos humanos; Prática profissional.

\begin{abstract}
The objective of this study was to describe a curricular experience based on the constructivist spiral in a discipline of the Physical Education course of the Federal University of Rio Grande do Sul (UFRGS), in the Unified Health System (SUS) context. In four meetings, each with four hours of classes, in two class groups, the corporal practices and physical activities (PCAF) in interface with SUS were thematized through the elaboration and attendance of problem cases. Inspired by everyday work situations, teams formed by students delimited and studied learning objectives based on emerging needs. Procedures such as network organization, matrix support technologies, unique therapeutic design, use of medical records, genogram, ecomap, among others, delimited the focus of the work of contextualizing the use of PCAF in case-problems. Considering the evaluation which were carried out, it is concluded that the proposal strengthened students' learning about SUS knowledge and practices.
\end{abstract}

Keywords: Curriculum; Problem-based learning; Unified Health System; Human resources; Professional practice.

\section{Introdução}

Passados mais de 40 anos da inserção de um profissional de Educação Física em serviço de saúde mental no Hospital Psiquiátrico São Pedro, em Porto Alegre, Rio Grande do Sul1; quase 30 anos de existência do Sistema Único de Saúde (SUS); além de dez anos da criação dos Núcleos de Apoio à Saúde da Família (NASF)², as experiências relevantes no campo da formação em Educação Física voltadas à saúde coletiva ainda são pontuais ${ }^{3-5}$. Por assim serem, ainda há, em algumas realidades, pro- fissionais de Educação Física que se formam com baixo ou nenhum conhecimento sobre o universo do SUS ${ }^{6}$.

Entre tantas iniciativas governamentais implementadas nos últimos anos para suprir lacunas na formação superior voltada para o Sistema Único de Saúde (SUS), destaca-se o Programa Nacional de Reorientação da Formação Profissional em Saúde (Pro-Saúde) como relevante indutor de reformulações curriculares nos mais diferentes cursos de formação superior em saúde no Brasil ${ }^{7}$.

Em função do compromisso assumido pela Univer- 
sidade Federal do Rio Grande do Sul (UFRGS) junto ao Pró-Saúde, várias inovações curriculares foram implantadas em diferentes cursos. No caso da Educação Física, foi criado um núcleo específico de disciplinas voltada para a ampliação do conhecimento pertinente ao SUS ${ }^{8}$. Uma das inovações foi a implantação da disciplina "Bases das Práticas Corporais e Saúde", que aborda as características, potencialidades e debilidades das PCAF para o uso em intervenções profissionais no campo da saúde ${ }^{8}$. Dada a centralidade dessa disciplina, o presente texto tem por objetivo relatar uma experiência curricular fundamentada no referencial da espiral construtivista ${ }^{9}$ em uma disciplina do curso de Educação Física da Universidade Federal do Rio Grande do Sul (UFRGS), no contexto do SUS.

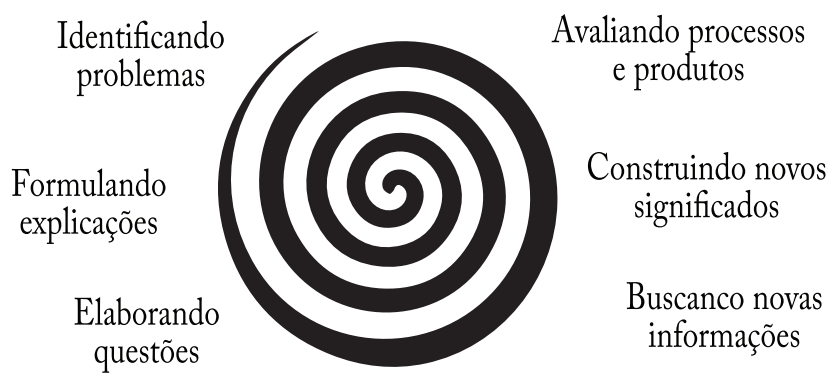

Figura 1 - Representação esquemática dos passos metodológicos para operacionalização da espiral construtivista. Adaptado de Lima ${ }^{8}$.

\section{Espiral construtivista e a localização da proposta}

A opção pelo referencial da espiral construtivista se deve a sua potencialidade em agregar ao processo de ensino-aprendizagem demandas que emergem nas situações do cotidiano de trabalho9. A espiral construtivista é uma metodologia problematizadora que surge a partir da releitura da aprendizagem baseada em problemas, de um currículo da medicina, sistematizada na dissertação de mestrado de Lima ${ }^{10}$. Nessa proposta, a autora congrega e reconhece: múltiplos caminhos possíveis na construção de um metaponto de vista (dialogia); os conhecimentos prévios e aqueles emergentes do contexto vivido (aprendizagem significativa); e o processo contínuo de pesquisas (metodologia científica) ${ }^{9}$. Esses elementos podem ser vislumbrados em outras experiências, tais como na formação de docentes na área da saúde ${ }^{11}$, na formação de preceptores e tutores no âmbito das residências multiprofissionais em saúde ${ }^{12,13}$, bem como na formação vinculada ao Ministério da Saúde de um curso de especialização em Ativação de Processos de Mudança na Formação Superior de Profissionais de Saúde ${ }^{14}$, contudo não há relatos mais sistemáticos sobre seu uso na graduação em Educação Física. A Figura 1 representa a organização da proposta.

De modo sintético, a operacionalização subdivide-se em: identificando problemas, com apreensão da problemática a ser investida; formulando hipóteses, que permite pensar empiricamente possibilidades de resolução, mas que culminam com dificuldades e lacunas; elaborando questões, que representam as necessidades de aprendizagem dos discentes, emergentes da etapa anterior; buscando novas informações, com busca de material que venha a aprimorar a abordagem inicial, reafirmando, repensando ou mesmo complementando-a; construindo novos significados, a partir do confronto entre os saberes prévios e as novas informações encontradas; e avaliando processo e produtos, que consiste em um processo que envolve autoavaliação, avaliação por pares e pelo facilitador.

A disciplina "Bases das Práticas Corporais na Saúde" é obrigatória e vem sendo oferecida regularmente, e simultaneamente, para os curso de licenciatura e bacharelado em Educação Física da UFRGS, sendo posicionada no oitavo semestre. De acordo com sua ementa, "aborda as características das diferentes práticas corporais sistematizadas para o uso em intervenções profissionais no campo da Saúde" e possui 60 horas-aulas, sendo um encontro semanal de quatro horas-aulas para duas turmas, uma matutina e outra vespertina, totalizando 18 encontros.

No segundo semestre de 2017, atividades baseadas na espiral construtivista foram, pela primeira vez, propostas para esta disciplina nas duas turmas, contando com a participação de de 52 alunos. A disciplina foi organizada em três momentos, que se desenvolveram da seguinte forma:

Os encontros relativos ao primeiro momento envolveram inicialmente uma pactuação com os alunos acerca da possibilidade de desenvolver, ou não, práticas pedagógicas a partir das metodologias ativas, considerando que, de modo geral, tal prática requer maior implicação. Houve um aprofundamento do que vem a ser tais métodos, já os utilizando. Temáticas relativas ao SUS, determinantes sociais da saúde, conceito de saúde e PCAFs também foram trabalhados. Esses encontros contaram com metodologias diversas de ensino, tais como discussão de textos, análise de entrevistas realizadas no próprio campus, busca de materiais na internet e na biblioteca, análise de vídeos, entre outros.

$\mathrm{O}$ segundo momento foi o que efetivamente contou 
com a fundamentação da espiral construtivista, seguindo suas etapas de operacionalização, descritas na sequência.

O terceiro momento envolveu uma visita aos cenários de práticas, em que os discentes puderam compreender o campo de atuação e promover diálogos contextualizados nos encontros em sala de aula. Esse processo ocorreu principalmente por meio de articulação com os programas de residência multiprofissional em saúde de Porto Alegre que contam com profissional de Educação Física.

\section{Operacionalização dos encontros}

Nas aulas relativas ao segundo momento, no qual as práticas pedagógicas foram organizadas com base na espiral construtivista ${ }^{8}$, cada uma das turmas foi dividida a critério do professor em quatro subgrupos (equipes) com cerca de seis integrantes. Basicamente, cada uma das equipes precisava se autogerir para contemplar dois objetos de intervenção: (1) dar conta de um caso-problema, inspirado em situações do cotidiano de trabalho, mas reconstituído por outra equipe e; (2) produzir um relato da experiência vivida, com ênfase na produção de um projeto terapêutico. Assim, a operacionalização das aulas e as responsabilidades das equipes se deu de acordo com a Figura 2.

No encontro 1 foram explicadas questões relativas à disciplina, tais como o trabalho a partir da elaboração da produção textual e do atendimento de casos. A produção textual se dividiu em três partes: (1) introdução, contendo uma contextualização da atividade; (2) formulação prévia, com elementos definidos no primeiro encontro, pela experiência inicial dos discentes e; (3) projeto terapêutico final, que possui a delimitação final acerca do caso no encerramento do terceiro encontro, a ser apresentado e compartilhado no último encontro. Desse modo, cada equipe produziu um texto para ser trabalhado no quarto encontro. Foi utilizada uma ficha que facilitava o monitoramento da produção textual e das demais atividades por meio de anotações realizadas pela própria equipe.

Por orientação direta do professor, os casos índices deveriam estar relacionados a alguma rede de atenção à saúde, inspirados em situações do cotidiano de trabalho, como também atravessados por diferentes determinantes (por exemplo, familiar, econômico, social, cultural). $\mathrm{Na}$ sequência, um membro da equipe representava o caso diante da turma, e era atendido por outra equipe, da qual cada integrante foi orientado a representar determinada categoria profissional (tais como fisioterapia, nutrição, psicologia), tendo como regra a necessidade de ao menos um deles como pertencente ao núcleo da Educação Física. Nesse momento, os discentes perceberam que no cenário de práticas do SUS, mesmo que a intenção seja atuar exclusivamente com PCAFs, é necessário mobilizar saberes que não se limitam ao núcleo da Educação Física, bem como desenvolver competências para lidar com outros instrumentos, tais como o registro em prontuário, a complexidade da re-

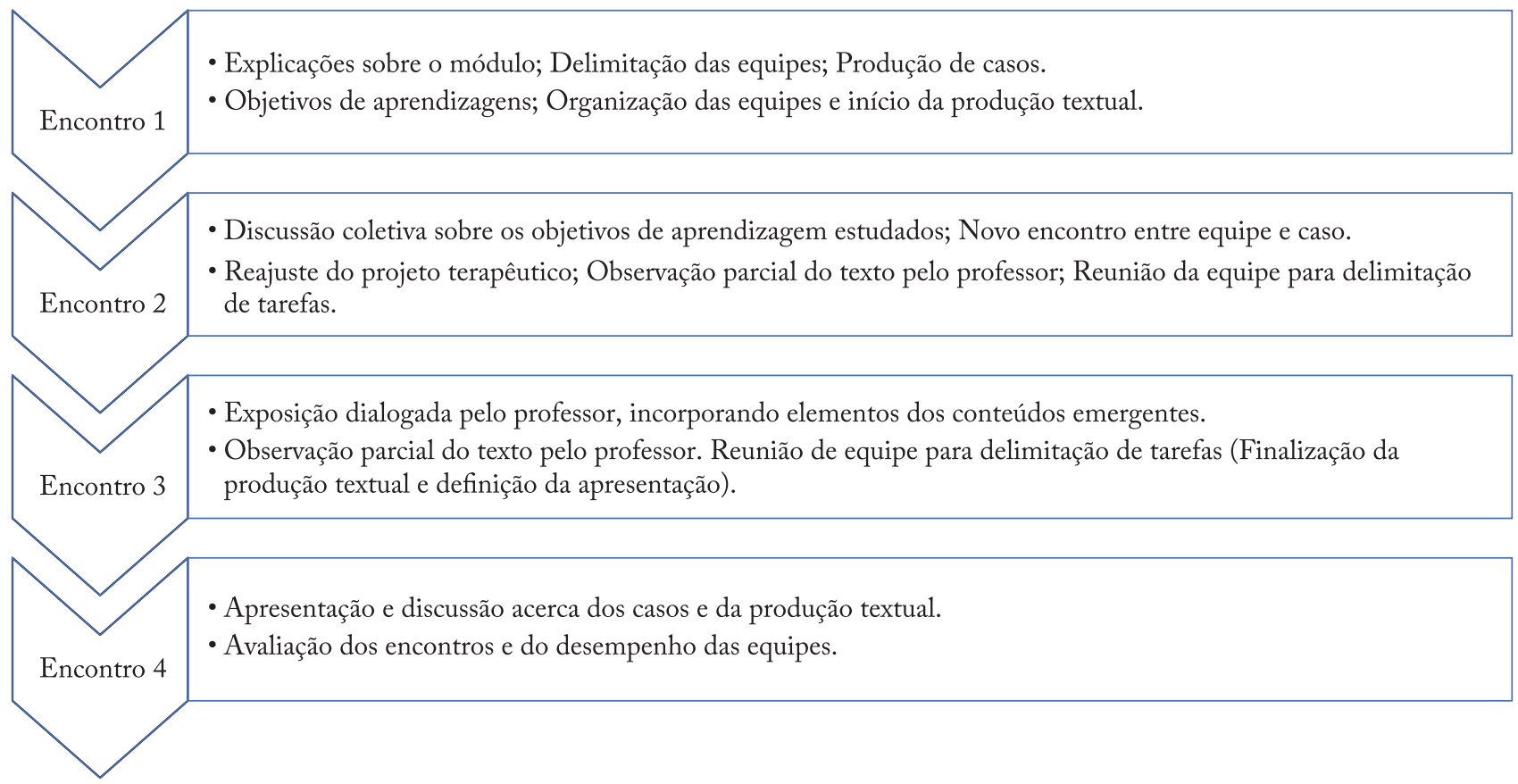

Figura 2 - Síntese das tarefas realizadas durante os encontros, cada um com duração de quatro horas. 
lação com outros profissionais e o exercício da escuta qualificada. A delimitação de objetivos de aprendizagem foi crucial na busca por referenciais que viessem a ajudá-los na construção daquela prática de cuidado.

Dada a natureza da atividade, as aulas se desenvolveram de tal modo que algumas demandas tiveram de ser contempladas fora da sala de aula. Após o primeiro encontro, era preciso delimitar quem se responsabilizaria por escrever determinada parte do texto e/ou pesquisar acerca de qual objetivo de aprendizagem. Com isso, já no encontro 2, foi realizada uma discussão coletiva levando em consideração a possibilidade de que alguma das temáticas estudada por uma equipe pudesse ser pertinente à construção do projeto terapêutico de outras. Na sequência, houve novo encontro com o caso, no qual as equipes incorporaram outros elementos baseados no que foi trabalhado durante a disciplina. Contudo, os discentes que representavam o caso foram orientados a apontar alguma intercorrência em relação aos encaminhamentos dados no primeiro encontro: foi encaminhado para um grupo de caminhada, mas não gostou da prática, por exemplo. Desse modo, os estudantes precisaram desenvolver a competência de lidar com esses imprevistos, bem como manejar o uso de referências para fundamentar o atendimento, reajustando a formulação prévia criada no encontro 1. Ao final, foi disponibilizado tempo para que as equipes se reunissem para discutir o caso e se organizassem para a produção do texto e para as aulas seguintes.

No encontro 3 ocorreu uma aula expositiva dialogada que visava a subsidiar a produção dos projetos terapêuticos. Foram trabalhadas temáticas como a organização do SUS, da Estratégia Saúde da Família (ESF), e do NASF; estratégias de intervenção, como apoio matricial e o projeto terapêutico singular; ferramentas de suporte aos projetos terapêuticos, como o uso de prontuários, elaboração de genograma e ecomapa. Metodologicamente, o diálogo se deu a partir do exemplo dos casos elaborados pelas equipes, o que facilitou a incorporação desse material nos textos.

O encontro 4 foi destinado inicialmente à discussão dos casos e da produção textual, que incluiu momentos de experimentação prática. Determinada equipe que havia atendido uma gestante (que integra a Rede Cegonha), por exemplo, apresentou algumas PCAFs passíveis de serem realizadas com este público, tais como exercícios respiratórios e relaxantes. Além disso, as equipes fizeram uso de tecnologias não específicas da Educação Física, tais como a visita domiciliar, o diá- logo intersetorial, genograma, ecomapa, prontuário e projeto terapêutico singular.

O processo de avaliação dos encontros, da aprendizagem dos alunos e do desempenho do professor se centrou em dois pontos: (1) assimilação dos conteúdos pelos estudantes por meio da produção textual, da participação durante e de uma autoavaliação da equipe e; (2) eficácia da metodologia ativa partir das respostas dos estudantes a questões lançadas em formulário disponibilizado via "Formulários Google". Quanto ao primeiro ponto, o principal critério foi a utilização do léxico do campo da saúde coletiva, sobretudo a articulação conceitual de tecnologias que circulam no âmbito do SUS, demonstrados principalmente pelas apresentações e textos produzidos - notamos alguns avanços por parte da maioria dos estudantes. E por meio das respostas obtidas em relação ao uso das metodologias ativas, foi possível perceber que a estratégia metodológica adotada favoreceu tal assimilação.

\section{Considerações finais}

A experiência desenvolvida mostrou-se relevante no sentido de evidenciar saberes e práticas com base em temáticas significativas. Trata-se de uma experiência interessante para fundamentar práticas de ensino em outras realidades, inclusive em disciplinas que não tematizem o SUS, mas que estejam implicadas em investir na busca por soluções de problemas próprios do cotidiano de trabalho. Por outro lado, são precisos reajustes, sobretudo no que se refere à carga horária de cada encontro, que geralmente não é de quatro horas-aulas, como na experiência aqui relatada. É igualmente determinante para uma experiência dessa natureza uma preparação teórica prévia e a incitação permanente à pesquisa de parte do docente, para que a tomada de decisão por parte dos alunos seja fundamentada.

Neste ínterim, propiciaram-se subsídios para repensar práticas fragmentadas e centradas em procedimentos, muitas vezes, descontextualizados em relação a situações do cotidiano de trabalho. A imbricação entre as aulas, processo colaborativo e conteúdos que emergiram do processo de pesquisa para dar conta do caso favorece maior aproximação com o cenário de práticas, considerando que provavelmente irão surgir demandas do serviço que necessitam de um trabalho colaborativo e uma busca de soluções por meio de pesquisas. É preciso destacar que disciplinas de cursos de formação superior que abordam as características das PCAFs e seu uso no campo da saúde requerem a aplicação de 
metodologias igualmente inovadoras, tal como a espiral construtivista descrita neste texto. No contexto específico desse curso, a proposta potencializou a aprendizagem de saberes e práticas relativos ao SUS.

\section{Conflito de interesse}

Os autores declaram não haver conflito de interesses.

\section{Financiamento}

O estudo contou com uma bolsa de doutorado Capes.

\section{Contribuição dos autores}

Oliveira BN, participou da experiência como estagiário docente vinculado ao Programa de Pós-Graduação em Ciências do Movimento Humano, do texto na concepção inicial e da redação. Damico JG, participou da experiência como professor da disciplina e supervisor de estágio docente, do texto na redação e da revisão crítica. Fraga AB, participou da experiência como professor da disciplina e orientador de estágio docente, do texto na redação e da revisão crítica.

\section{Referências}

1. Santos DF. Educação Física no Hospital Psiquiátrico São Pedro: uma experiência de familiarização e estranhamento nas entrelinhas dos relatos de estágio. [dissertação de mestrado]. Porto Alegre: Universidade Federal do Rio Grande do Sul; 2016.

2. Ministério da Saúde. Portaria no 154 de 24 de janeiro de 2008. Cria os Núcleos de Apoio à Saúde da Família-NASF. In: Diário Oficial da União [online], 2008. [citado 2018 mai 18]. Disponível em: http://bvsms.saude.gov.br/bvs/ saudelegis/gm/2008/prt0154_24_01_2008.html

3. Fraga AB, Carvalho YMD, Gomes IM. Políticas de formação em educação física e saúde coletiva. Trab Educ Saúde (Online). 2012;10(3):367-86.

4. Loch MR. Abordando Saúde Coletiva no curso de bacharelado em Educação Física: Relato de experiência. Rev Bras Ativ Fis Saúde. 2016;21(3):285-90.

5. Silva J, Andrade M, Brito A, Hardman C, Oliveira E, Barros M. Atividade física e saúde mental: uma experiência na formação inicial em Educação Física. Rev Bras Ativ Fis Saúde. 2014;19(1):133-40.
6. Manske GS, Oliveira D. A formação do profissional de Educação Física e o Sistema Único de Saúde. Motriv. 2017; 29(52):191-210.

7. Batista SHSS, Jansen B, Assis EQ, Senna MIB, Cury GC. Formação em Saúde: reflexões a partir dos Programas PróSaúde e PET-Saúde. Interface (Botucatu). 2015;19(Suppl 1):743-52.

8. Comgrad. Comissão de Graduação. Projeto pedagógico do curso bacharelado em Educação Física. Plano Pedagógico do Curso de Educação Física - Habilitação Bacharelado. Universidade Federal do Rio Grande do Sul, 2017. [citado 2018 mai 18]. Disponível em: https://www.ufrgs.br/esefid/ Arquivos/COMGRAD_EFI/ppc_bacharelado.pdf

9. Lima VV. Espiral construtivista: uma metodologia ativa de ensino-aprendizagem. Interface (Botucatu). 2017;21(61):421-34.

10. Lima VV. Learning issues raised by students during PBL tutorials compared to curriculum objectives [dissertation]. Chicago: University of Illinois at Chicago; 2001.

11. Macedo KDS, Acosta BS, Silva EB, Souza NS, Beck CLC, Silva KKD. Metodologias ativas de aprendizagem: caminhos possíveis para inovação no ensino em saúde. Esc Anna Nery. 2018;22(3):e20170435.

12. Meneses LBA, Leie VS, Pereira AJ, Rocha RCS, Fernandes MCV. Vivendo a formação de preceptores e tutores: uma experiência refletida. In: Ceccim RB, Meneses LBA, Soares VL, Pereira AJ, Meneses JR, Rocha RCS et al. (eds). Formação de Formadores para Residências em Saúde: corpo docente-assistencial em experiência viva. Porto Alegre: Rede UNIDA; 2018. p. 61-74.

13. Camargo FC, Garcia LAA, Walsh IAP, Emílio MM, Coelho VHM, Pereira GA. Formação para o trabalho na estratégia saúde da família: experiência da residência multiprofissional em saúde. Rev Enferm Atenção à Saúde. 2018;7(1):190-99.

14. Lima VV, Feuerwerker LCM, Padilha RQ, Gomes R, Hortale VA. Ativadores de processos de mudança: uma proposta orientada à transformação das práticas educacionais e da formação de profissionais de saúde. Cienc Saúde Colet. 2015;20(1):279-88. 